EPJ manuscript No.

(will be inserted by the editor)

\title{
Smoothening Transition of a Two-Dimensional Pressurized Polymer Ring
}

\author{
Emir Haleva and Haim Diamant \\ School of Chemistry, Raymond \& Beverly Sackler Faculty of Exact Sciences, Tel Aviv University, Tel Aviv 69978, Israel \\ Received: date / Revised version: date
}

\begin{abstract}
We revisit the problem of a two-dimensional polymer ring subject to an inflating pressure differential. The ring is modeled as a freely jointed closed chain of $N$ monomers. Using a Flory argument, mean-field calculation and Monte Carlo simulations, we show that at a critical pressure, $p_{\mathrm{c}} \sim N^{-1}$, the ring undergoes a second-order phase transition from a crumpled, random-walk state, where its mean area scales as $\langle A\rangle \sim N$, to a smooth state with $\langle A\rangle \sim N^{2}$. The transition belongs to the mean-field universality class. At the critical point a new state of polymer statistics is found, in which $\langle A\rangle \sim N^{3 / 2}$. For $p \gg p_{\mathrm{c}}$ we use a transfer-matrix calculation to derive exact expressions for the properties of the smooth state.
\end{abstract}

PACS. 36.20.Ey Macromolecules and polymer molecules: Conformation (statistics and dynamics) 05.40.Fb Random walks and Levy flights - 64.60.-i General studies of phase transitions

\section{Introduction}

Considerable theoretical efforts were directed during the 1980s and 1990s at random polymer rings constrained to a plane, both as a fundamental problem of statistical mechanics 1, 2, 3, 4,5] and as a highly idealized model for membrane vesicles 6, 7 8, $9,10,11,12,13,14$. In addition, there was much interest in transitions between crumpled and smooth states of membranes 15 16.

Two bodies of work, in particular, concerned pressur' ized two-dimensional (2D) rings. Fisher et al. studied both inflated and deflated, closed, 2D self-avoiding walks using Monte Carlo (MC) simulations and scaling analysis 6.7. 8. For a finite ring of $N$ monomers, subject to an inflating pressure differential $p>0$, three regimes were found: (i) ' a weak-inflation crumpled regime for $0<p N^{2 \nu} \lesssim 1(\nu=$ $3 / 4$ being the swelling exponent of $2 \mathrm{D}$ self-avoiding walks), where the mean area scales as $\langle A\rangle \sim N^{2 \nu} f_{A}\left(p N^{2 \nu}\right)$; (ii) a strong-inflation crumpled regime for $1 \lesssim p N^{2 \nu} \ll N^{2 \nu-1}$, where the same scaling holds but the scaling function $f_{A}$ becomes a power law; and (iii) a smooth regime for $p N^{2 \nu} \gtrsim N^{2 \nu-1}$, where $\langle A\rangle \sim N^{2}$. The crossovers between these regimes are gradual with no phase transitions. In the thermodynamic limit, defined as $N \rightarrow \infty$ and $p \rightarrow 0$ such that $p N^{2 \nu}$ is finite, the range of the scaling regime (ii) in the case of self-avoiding walks $(\nu=3 / 4)$ becomes infinite. Note, however, that in the random-walk case $(\nu=1 / 2)$ this regime disappears; we will show below that for random walks regime (ii) turns, in fact, into a second-order phase transition between regimes (i) and (iii).

The second body of works consists of analytical studies of closed, pressurized, 2D random walks, first presented by Rudnick and Gaspari 9 10,11. In this model the poly- mer comprises a set of Gaussian springs of fixed elastic constant and no bending rigidity. At a critical pressure $p_{\mathrm{c}} \sim N^{-1}$ the mean area was found to diverge (i.e., the ring inflates to infinity). This divergence, obviously, is made possible by the extensibility of the chain, i.e., the ability of the springs in this model to be infinitely stretched.

In the current work we revisit the Rudnick-Gaspari model while imposing inextensibility of the chain, as is actually appropriate for real polymers or vesicles. This constraint changes the infinite inflation at $p=p_{\mathrm{c}}$ into a second-order phase transition between a crumpled, randomwalk state and a smooth one. The model is defined in Section 2 We begin the investigation in Section 3 with a simple Flory argument which, nevertheless, yields the correct physics. In Section 4 we present a mean-field calculation which accurately captures the behavior below and at the transition. In Section [5]we derive exact expressions for the behavior at $p \gg p_{\mathrm{c}}$ using a transfer-matrix technique. The behavior found in Sections [35] is verified in Section [6] by MC simulations. Finally, the results are summarized and discussed in Section $\square$

\section{Model}

The system under consideration is illustrated in Fig. 1 A closed, two-dimensional, freely jointed chain of $N$ monomers is subject to an inflating 2D pressure differential $p>0$ between its interior and exterior. The monomers are connected by rigid links of length $l$. We define $l \equiv 1$ as the unit length and the thermal energy $k_{\mathrm{B}} T$ as the unit energy. (Thus, $p$ is measured in units of $k_{\mathrm{B}} T / l^{2}$.) The chain 
is ideal, i.e., there are no interactions between monomers. (Effects related to self-avoidance will be briefly discussed in Sections 3 and 7 ) In addition, no bending rigidity is taken into account, i.e., the chain is freely jointed. A configuration of the ring is defined by a set of $2 \mathrm{D}$ vectors $\left\{\mathbf{r}_{j}\right\}_{j=0 \ldots N}$ specifying the positions of the monomers. The condition that the chain be closed is expressed by $\mathbf{r}_{0}=\mathbf{r}_{N}$.

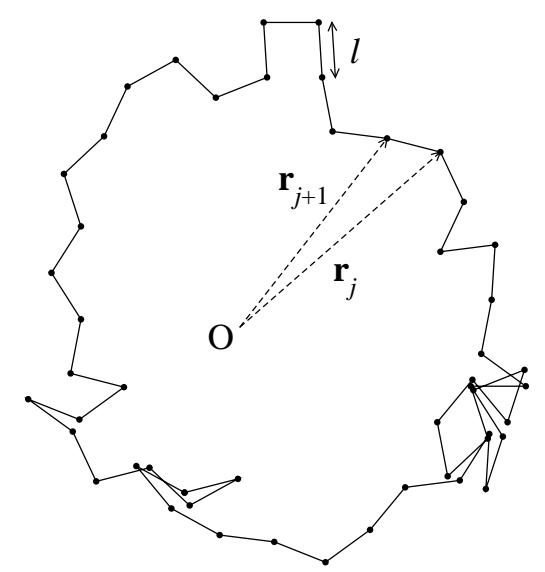

Fig. 1. Schematic illustration of the ring and its parameters.

The probability of a specific configuration is

$$
P\left(\left\{\mathbf{r}_{j}\right\}, p\right) \propto e^{p A\left[\left\{\mathbf{r}_{j}\right\}\right]} \prod_{j=1}^{N} \delta\left(\left|\mathbf{r}_{j}-\mathbf{r}_{j-1}\right|-1\right)
$$

where $A$ is the area enclosed by the ring. As in previous works [2, 3, 10,11, we take $A$ as the algebraic area rather than the geometrical one,

$$
A\left[\left\{\mathbf{r}_{j}\right\}\right]=\frac{1}{2} \sum_{j=1}^{N}\left(\mathbf{r}_{j-1} \times \mathbf{r}_{j}\right) \cdot \hat{\mathbf{z}}
$$

where $\hat{\mathbf{z}}$ is a unit vector perpendicular to the plane of the ring. This area may take both positive and negative values. At zero pressure both signs are equally favorable and the mean area must vanish. At high pressures the probability of configurations with negative $A$ is exponentially small in $p|A|$, and whether one takes the algebraic or geometrical area will become statistically insignificant. (We shall further discuss this assumption in Section [7)

Using Eqs. (11) and (2), we write the partition function of the ring as

$$
Z(p, N)=\int \prod_{j=1}^{N} d \mathbf{r}_{j} e^{\frac{1}{2} p\left(\mathbf{r}_{j-1} \times \mathbf{r}_{j}\right) \cdot \hat{\mathbf{z}}} \delta\left(\left|\mathbf{r}_{j}-\mathbf{r}_{j-1}\right|-1\right)
$$

with $\mathbf{r}_{0}=\mathbf{r}_{N}$.

\section{Flory Argument}

We begin the analysis with a simple Flory argument 17] which captures most of the physics to be more rigorously treated in the following sections. The free energy of the ring (in units of $k_{\mathrm{B}} T$ ) is expressed as a function of $R$, the radius of the statistical cloud of monomers (i.e., the rootmean-square radius of gyration). We divide it into three terms,

$$
\begin{aligned}
F(R) & =F_{\text {el }}+F_{\text {inext }}+F_{p} \\
F_{\text {el }} & \sim R^{2} / N \\
F_{\text {inext }} & \sim R^{4} / N^{3} \\
F_{p} & \sim-p R^{2} .
\end{aligned}
$$

The elastic term, $F_{\mathrm{el}}$, is the usual entropic-spring free energy of a Gaussian chain [17. The second term is the leading non-Gaussian correction due to the inextensibility of the chain [see Appendix, Eq. A.5], needed here to stabilize the ring against infinite expansion. The last term is the pressure contribution, where the mean area of the ring is taken as proportional to $R^{2} 45$.

Equation (4) has the form of a Landau free energy, describing a second-order transition at $p=p_{\mathrm{c}} \sim N^{-1}$. Since the critical pressure depends so strongly on system size, we use hereafter the rescaled pressure $\hat{p} \equiv p / p_{\mathrm{c}} \sim p N$, and define the thermodynamic limit as $N \rightarrow \infty$ and $p \rightarrow 0$ such that $\hat{p}$ is finite. For $\hat{p}<1 F_{\text {inext }}$ is negligible, and $R$ has a Gaussian distribution with $\left\langle R^{2}\right\rangle \sim N(1-\hat{p})^{-1}$. For $\hat{p}>1$ we have $R^{2} \sim N^{2}(\hat{p}-1)$. Thus, defining an order parameter $M=R / N$, we find in the thermodynamic limit

$$
M \sim \begin{cases}0 & \hat{p}<1 \\ (\hat{p}-1)^{\beta}, \quad \beta=1 / 2 & \hat{p}>1\end{cases}
$$

At the critical point itself $F=F_{\text {inext }} \sim R^{4} / N^{3}$, and $R$ has a non-Gaussian distribution with

$$
\left\langle R^{2}(\hat{p}=1)\right\rangle \sim N^{2 \nu_{\mathrm{c}}}, \quad \nu_{\mathrm{c}}=3 / 4
$$

Note that the competition between $F_{\mathrm{el}}$ and $F_{p}$, leading to the second-order transition, is unique to $2 \mathrm{D}$. In addition, when an excluded-volume term, $F_{\text {ev }} \sim N^{2} / R^{2}$, is added to the free energy, Eq. (4), the transition is removed. This agrees with previous studies of self-avoiding rings 6,7,8, which did not report any phase transition upon increasing pressure.

\section{Mean-Field Theory}

In this section we calculate the partition function of the freely jointed ring, Eq. (3), by relaxing the rigid deltafunction constraints on link lengths into harmonic potentials. The spring constant $\lambda$ of the links is chosen so as to make the root-mean-square length of a link equal to $l=1$. This type of approximation, first suggested by Harris and Hearst [18, was successfully employed in studies of the Karatky-Porod worm-like-chain model [18 19], where 
it was shown to be equivalent to a mean-field assumption (for the field conjugate to the rigid link-length constraints). The partition function contains now only Gaussian terms and, therefore, can be calculated exactly,

$$
\begin{aligned}
Z(p, N, \lambda) & =\int \prod_{j=1}^{N} d \mathbf{r}_{j} e^{\frac{1}{2} p\left(\mathbf{r}_{j-1} \times \mathbf{r}_{j}\right) \cdot \hat{\mathbf{z}}-\lambda\left(\mathbf{r}_{j}-\mathbf{r}_{j-1}\right)^{2}} \\
& =\frac{1}{\lambda^{N}} \frac{N p}{4 \lambda \sin \left(\frac{N p}{4 \lambda}\right)} .
\end{aligned}
$$

(The spring constant $\lambda$ is in units of $k_{\mathrm{B}} T / l^{2}$.) Details of the calculation can be found in Ref. 10. This result can be obtained also by analogy to the quantum propagator of a charged particle in a magnetic field [20. The mean area is obtained by differentiation with respect to $p$,

$$
\langle A(p, N, \lambda)\rangle=\frac{\partial \ln Z}{\partial p}=\frac{1}{p}-\frac{N \cot \left(\frac{N p}{4 \lambda}\right)}{4 \lambda} .
$$

For $\lambda=1$ Eq. (8) is the same as the result obtained by Rudnick and Gaspari 9,10,11, exhibiting a divergence at

$$
p_{\mathrm{c}}=4 \pi / N \text {. }
$$

Yet, in our case $\lambda$ is not fixed but is to be determined self-consistently to ensure the softened inextensibility constraint. It is clear that, as the pressure increases, the springs must become stiffer to satisfy this constraint. To impose the constraint, we calculate the mean-square link length and set it to 1 ,

$$
\begin{aligned}
\left\langle\left(\mathbf{r}_{j}-\mathbf{r}_{j-1}\right)^{2}\right\rangle & =-\frac{1}{N} \frac{\partial \ln Z}{\partial \lambda} \\
& =\frac{1}{\lambda}+\frac{p}{N \lambda}\left(\frac{1}{p}-\frac{N \cot \left(\frac{N p}{4 \lambda}\right)}{4 \lambda}\right)=1
\end{aligned}
$$

thus obtaining a transcendental equation for $\lambda(p, N)$. Equations (8) and (10) are combined to yield a simpler expression for $\langle A\rangle$ as a function of $\lambda$,

$$
\langle A(p, N, \lambda)\rangle=\frac{N(\lambda-1)}{p} .
$$

Numerical solution of Eq. (10) for $\lambda$ [in the range $\lambda>$ $N p /(4 \pi)$ ] and substitution of the result in Eq. (11) yield the mean area as a function of $p$ and $N$. (See dashed curves in Fig. 2

For very low pressures, $p \ll p_{\text {c }}$, we expand Eq. (8) to first order in $p$ to get

$$
\left\langle A\left(p \ll p_{\mathrm{c}}, N\right)\right\rangle=\frac{1}{48} N^{2} p,
$$

i.e., a linear dependence on $p$ as expected from linear response. (Recall that at $p=0$ the mean algebraic area vanishes.)

For higher pressure we obtain a good approximation for $\lambda(p, N)$ in the limit $N \gg 1$ by substituting in Eq. (10)

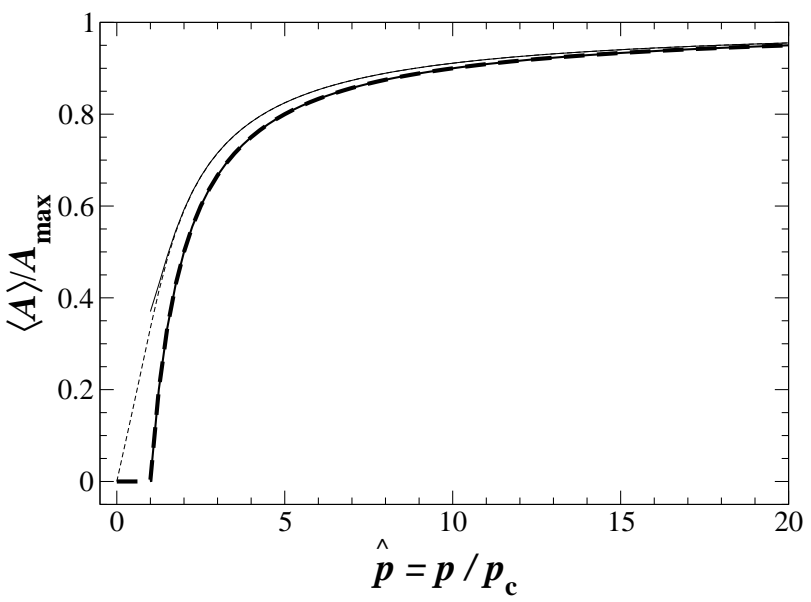

Fig. 2. The mean area in units of $A_{\max } \sim N^{2}$ as a function of the rescaled pressure (in units of $p_{\mathrm{c}} \sim N^{-1}$ ) as obtained from the mean-field approximation. The dashed curves are calculated numerically using Eqs. (10) and (11), whereas the solid curves (calculated only for $\hat{p} \geq 1$ ) present the approximation given by Eq. (15). Calculations were performed for $N=10$ (thin curves) and $N=10^{5}$ (thick curves).

$\cot [N p /(4 \lambda)] \simeq[N p /(4 \lambda)-\pi]^{-1}$. This gives

$\lambda(\hat{p}, N \gg 1) \simeq \frac{\hat{p}+1+\frac{1}{N}+\sqrt{(\hat{p}-1)^{2}+\frac{2}{N}(\hat{p}+1)+\frac{1}{N^{2}}}}{2}$,

where $\hat{p} \equiv p / p_{\mathrm{c}}=p N /(4 \pi)$ is the rescaled pressure. In the thermodynamic limit Eq. (13) reduces to the continuous but nonanalytic function

$$
\lambda(\hat{p}, N \rightarrow \infty)= \begin{cases}1 & \hat{p}<1 \\ \hat{p} & \hat{p}>1\end{cases}
$$

Equation (13) should be regarded as an asymptotic expression in the limit $N p /(4 \lambda) \rightarrow \pi$, which turns out to be valid for any $p \ll p_{\mathrm{c}}$. (A Taylor expansion around this point fails because of the nonanalyticity inferred above.) Substituting Eq. (13) in Eq. (11) yields an approximate expression for $\langle A\rangle$ as a function of $\hat{p}$ and $N$,

$$
\begin{aligned}
& \langle A(\hat{p} \nless 1, N \gg 1)\rangle \simeq \\
& \frac{N^{2}}{4 \pi} \frac{\hat{p}-1+\frac{1}{N}+\sqrt{(\hat{p}-1)^{2}+\frac{2}{N}(\hat{p}+1)+\frac{1}{N^{2}}}}{2 \hat{p}} .
\end{aligned}
$$

In the thermodynamic limit, the behavior of $\langle A\rangle$ around and above the critical pressure is obtained from Eq. (15) as

$$
\langle A\rangle= \begin{cases}\frac{N}{4 \pi} \frac{1}{\hat{p}(1-\hat{p})} \stackrel{\hat{p} \rightarrow 1^{-}}{\longrightarrow} \frac{N}{4 \pi} \frac{1}{1-\hat{p}} & 1-\hat{p} \gg N^{-1 / 2} \\ \frac{N^{3 / 2}}{4 \pi} & |1-\hat{p}| \ll N^{-1 / 2} \\ \frac{N^{2}}{4 \pi} \frac{\hat{p}-1}{\hat{p}} \stackrel{\hat{p} \rightarrow 1^{+}}{\longrightarrow} \frac{N^{2}}{4 \pi}(\hat{p}-1) & \hat{p}-1 \gg N^{-1 / 2}\end{cases}
$$


revealing a continuous (second-order) transition. Below the transition we get the same behavior as in the RudnickGaspari model $9.10,\langle A\rangle \sim N(1-\hat{p})^{-1}$. Yet, due to the inextensibility in our model, the increase of $\langle A\rangle$ as $\hat{p} \rightarrow 1^{-}$breaks at $|1-\hat{p}| \sim N^{-1 / 2}$. In the transition region, $|1-\hat{p}| \ll N^{-1 / 2}$, which shrinks to a point in the thermodynamic limit, we find $\langle A\rangle \sim N^{2 \nu_{\mathrm{c}}}, \nu_{\mathrm{c}}=3 / 4$. Above the transition the ring reaches a smooth state with $\langle A\rangle \sim N^{2}(\hat{p}-1) / \hat{p}$. All of these results agree with the findings of the Flory argument presented in Section 3 once we identify $\langle A\rangle \sim\left\langle R_{\mathrm{g}}^{2}\right\rangle$. As $p$ is increased to infinity, $\langle A\rangle$ tends, as it should, to

$$
\langle A(\hat{p} \rightarrow \infty, N)\rangle=A_{\max }=\frac{N^{2}}{4 \pi},
$$

which is the area of a circle of perimeter $N$.

Figure 2 shows the dependence of $\langle A\rangle$ on $\hat{p}$ for $N=10$ and $10^{5}$ calculated both from the numerical solution of Eqs. (10) and (11), and using the approximate expression (15). For large $N$ the critical behavior becomes apparent, with a transition between two distinct states - one in which $\langle A\rangle \sim N$ and, hence, in units of $A_{\max } \sim N^{2}$, the mean area vanishes for $N \rightarrow \infty$, and another with a mean area proportional to $A_{\max } \sim N^{2}$. As can be seen in Fig. 2 the approximate expression (15) is practically indistinguishable from the numerical solution for $\hat{p} \gtrsim 1$, even for small $N$.

The compressibility (defined with respect to the reduced pressure $\hat{p}$ ) is obtained from Eq. (16) as

$$
\kappa=\frac{1}{A} \frac{\partial A}{\partial \hat{p}} \stackrel{\hat{p} \rightarrow 1^{ \pm}}{\longrightarrow}|\hat{p}-1|^{-\gamma}, \quad \gamma=1 .
$$

At the critical point itself the compressibility diverges as

$$
\kappa(\hat{p}=1)=\frac{1}{2} N^{1 / 2} .
$$

To calculate the mean-square radius of gyration, $\left\langle R_{\mathrm{g}}^{2}\right\rangle=$ $N^{-1} \sum \mathbf{r}_{j}^{2}$ (where $\mathbf{r}_{j}$ are measured with respect to the center of mass), we add a $h \sum \mathbf{r}_{j}^{2}$ term to the Hamiltonian of Eq. (7) and differentiate the resulting partition function with respect to $h$. This yields

$$
\begin{aligned}
\left\langle R_{\mathrm{g}}^{2}(p, N, \lambda)\right\rangle & =\left.\frac{1}{N} \frac{\partial \ln Z(p, N, \lambda, h)}{\partial h}\right|_{h=0}= \\
& =\frac{4 \lambda-N p \cot \left(\frac{N p}{4 \lambda}\right)}{N p^{2}}=\frac{4 \lambda}{N p}\langle A\rangle .
\end{aligned}
$$

For $\hat{p} \ll 1$, combining this result with Eqs. (12) and (14), we get $\left\langle R_{\mathrm{g}}^{2}\right\rangle=N / 12$, which is the well known result for the mean-square radius of gyration of a Gaussian ring (e.g., 17). For large pressures, $\hat{p} \geq 1$, we have from Eq. (14) $\lambda \stackrel{N \rightarrow \infty}{\longrightarrow} \hat{p}=p N /(4 \pi)$, thereby recovering the relation for an average circle, $\pi\left\langle R_{\mathrm{g}}^{2}\right\rangle=\langle A\rangle$.

Figure 3 shows the dependence of $\left\langle R_{\mathrm{g}}^{2}\right\rangle$ on $N$ at fixed pressure. The data were obtained by substituting the numerical solution for $\lambda$ [Eq. (13)] in Eq. (20). The scaling of $\left\langle R_{\mathrm{g}}^{2}\right\rangle$ changes at the critical point $N_{\mathrm{c}}=4 \pi / p$. Below the critical point, $N<N_{\mathrm{c}},\left\langle R_{\mathrm{g}}^{2}\right\rangle \sim N$, as in a Gaussian chain. Above it $\left\langle R_{\mathrm{g}}^{2}\right\rangle \sim N^{2}$, as in a stretched chain. As $p$ decreases, the transition becomes sharper. At exactly $N=N_{\mathrm{c}}\left\langle R_{\mathrm{g}}^{2}\right\rangle$ scales as $N^{3 / 2}$. Thus, the analysis of $\left\langle R_{\mathrm{g}}^{2}\right\rangle$ yields the same scaling with $N$ as obtained for $\langle A\rangle$.

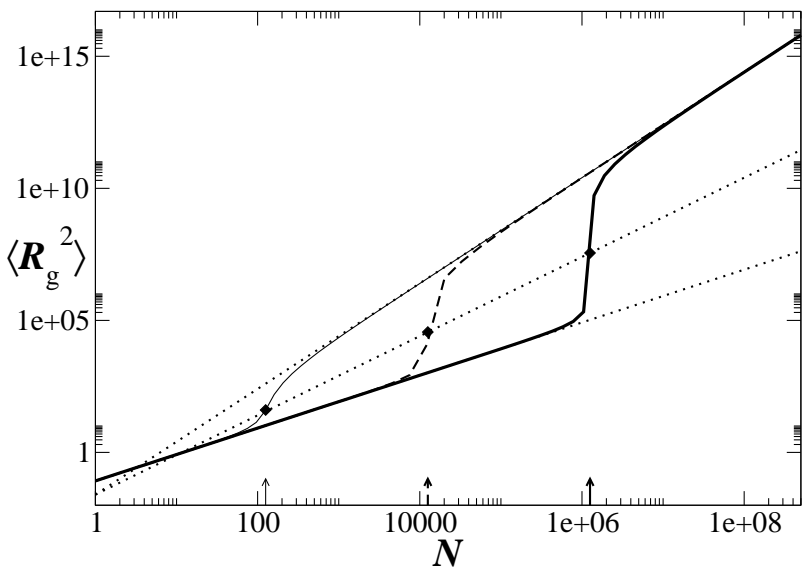

Fig. 3. Mean-square radius of gyration as a function of $N$ at fixed values of $p=10^{-5}, 10^{-3}$ and $10^{-1}$ (thick, dashed and thin curves, respectively) as obtained from the mean-field theory. For $N<N_{c}=4 \pi / p$ (marked with arrows) $\left\langle R_{\mathrm{g}}^{2}\right\rangle \sim N$, while for $N>N_{c}\left\langle R_{\mathrm{g}}^{2}\right\rangle \sim N^{2}$. At the critical point (diamonds) $\left\langle R_{\mathrm{g}}^{2}\right\rangle$ is proportional to $N^{3 / 2}$. The dotted lines show, from bottom to top, the dependencies for an unpressurized ring $\left(\left\langle R_{\mathrm{g}}^{2}\right\rangle=N / 12\right)$, for a ring at the critical pressure $\left[\left\langle R_{\mathrm{g}}^{2}\right\rangle=N^{3 / 2} /\left(4 \pi^{2}\right)\right]$ and for a stretched circle $\left[\left\langle R_{\mathrm{g}}^{2}\right\rangle=N^{2} /\left(4 \pi^{2}\right)\right]$.

\section{Transfer-Matrix Formulation}

Since the interactions between all adjacent monomers are identical, including the one between the first and the $N$ th, the partition function may be rewritten in the following transfer-matrix form:

$$
\begin{aligned}
& Z(p, N)=\operatorname{Tr}_{\left\{\mathbf{r}_{j}\right\}} \prod_{j=1}^{N} T\left(\mathbf{r}_{j-1}, \mathbf{r}_{j}\right), \quad \mathbf{r}_{0}=\mathbf{r}_{N} \\
& T\left(\mathbf{r}, \mathbf{r}^{\prime}\right)=e^{\frac{p}{2}\left(\mathbf{r} \times \mathbf{r}^{\prime}\right) \cdot \hat{\mathbf{z}}} \delta\left(\left|\mathbf{r}-\mathbf{r}^{\prime}\right|-1\right) .
\end{aligned}
$$

Solution of the associated eigenvalue problem,

$$
\mu \Psi(\mathbf{r})=\int d \mathbf{r}^{\prime} T\left(\mathbf{r}, \mathbf{r}^{\prime}\right) \Psi\left(\mathbf{r}^{\prime}\right),
$$

(in particular, finding the two eigenvalues $\mu$ of largest absolute value) will yield the exact solution of the model.

Two properties of the operator $T$ are readily noticed: it is non-Hermitian, $T\left(\mathbf{r}, \mathbf{r}^{\prime}\right) \neq T\left(\mathbf{r}^{\prime}, \mathbf{r}\right)$, and it is rotationalinvariant. To exploit the invariance to rotations, we change to polar coordinates, $\mathbf{r}=(\rho, \varphi)$, and separate variables as $\Psi(\mathbf{r})=\chi(\rho) e^{i m \varphi}$, where $m=0, \pm 1, \pm 2, \ldots$ to maintain 
periodicity in $\varphi$. Equation (22) can then be integrated over angles to give

$$
\begin{aligned}
& \mu \chi(\rho)=\int_{\rho-1}^{\rho+1} d \rho^{\prime} \tilde{T}\left(\rho, \rho^{\prime}\right) \chi\left(\rho^{\prime}\right) \\
& \tilde{T}\left(\rho, \rho^{\prime}\right)=\frac{2 \cosh \left(p \rho \rho^{\prime} \sqrt{1-\Delta^{2}} / 2+i m \cos ^{-1} \Delta\right)}{\rho \sqrt{1-\Delta^{2}}} \\
& \Delta\left(\rho, \rho^{\prime}\right)=\frac{\rho^{2}+\rho^{\prime 2}-1}{2 \rho \rho^{\prime}},
\end{aligned}
$$

thus reducing the original operator to the one-dimensional operator $\tilde{T}$.

Unfortunately, we have not been able to diagonalize $\tilde{T}$ for any $p$. For $p \gg p_{\mathrm{c}}$, nevertheless, the ring has stretched configurations, and we can assume that the distances of all monomers from the center of mass are much larger than the link length, $\rho \gg 1$. We expand $\chi\left(\rho^{\prime}\right)$ around $\rho$ to zeroth order, change variables according to $\rho^{\prime}=\rho+\sin \theta$, and integrate over $\theta$ to get

$$
\mu^{(0)} \chi(\rho)=2 \pi I_{0}(p \rho / 2) \chi(\rho),
$$

where $I_{0}$ is the zeroth-order modified Bessel function of the first kind. Thus, at this order of approximation, the eigenfunctions have the form $\chi_{k}^{(0)}(\rho)=\left[N /\left(2 \pi \rho_{k}\right)\right]^{1 / 2} \delta(\rho-$ $\left.\rho_{k}\right)$ with a continuous spectrum of eigenvalues. The spectrum is bounded from above by $\mu_{\max }^{(0)}=2 \pi I_{0}\left(p \rho_{\max } / 2\right)=$ $2 \pi I_{0}[p N /(4 \pi)]$, where $\rho_{\max }=N /(2 \pi)$ is the radius of a perfect circle of perimeter $N$. (The value of $\rho_{\max }$ can also be obtained from the condition that $\int|\chi(\rho)|^{2} d \rho \geq 1$, i.e., that going from the center of mass outward, one must cross the ring at least once.)

Within the zeroth-order approximation, and in the thermodynamic limit, the partition function is given by

$$
Z^{(0)}(p, N)=\left[\mu_{\max }^{(0)}\right]^{N}=\left[2 \pi I_{0}(p N / 4 \pi)\right]^{N} .
$$

This result has a straightforward interpretation. As shown in the Appendix [Eq. A.1 ], it is identical to the partition function of a $2 \mathrm{D}$, open, freely jointed chain subject to a tensile force $f=p N /(4 \pi)$. This force is just the tension associated with a Laplace pressure $p$ acting on a circle of radius $\rho_{\max } \sim N$. The mean area is obtained from Eq. (25) as

$$
\langle A(\hat{p}, N)\rangle=\frac{\partial \ln Z}{\partial p}=\frac{N^{2}}{4 \pi} \frac{I_{1}(\hat{p})}{I_{0}(\hat{p})},
$$

which saturates, as expected, to $A_{\max }=N^{2} /(4 \pi)$ as $p \rightarrow$ $\infty$. The approach to saturation is given by

$$
\langle A(\hat{p} \gg 1)\rangle / A_{\max } \simeq 1-\frac{1}{2 \hat{p}}
$$

which corrects the mean-field prediction, Eq. (16), by a factor of 2 .

\section{Monte Carlo Simulations}

Since our Flory argument and mean-field theory may fail near the critical point, we conducted Monte Carlo simulations to obtain the mean area $\langle A\rangle$, mean-square radius of gyration $\left\langle R_{\mathrm{g}}^{2}\right\rangle$ and mean-square area fluctuation $\left\langle\Delta A^{2}\right\rangle$ as a function of pressure $p$ for different ring sizes $N$.

\subsection{Numerical Scheme}

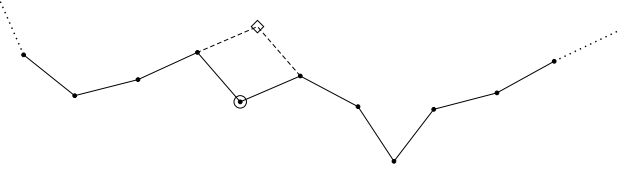

Fig. 4. Schematic view of a section of the simulated polygon. A randomly chosen vertex (marked by a circle) can be moved to a single position only (marked by a diamond) so as to maintain the lengths of the two links attached to it constant. The area enclosed by the resulting rhomb is the difference in total polygon area for the given step.

We consider a polygon of $N$ equal edges, defined by the $2 \mathrm{D}$ coordinates of its vertices. An off-lattice simulation is used, i.e., the positions of the vertices are defined in continuous space. At each step a random vertex is chosen and moved to the only other position that satisfies the edge-length constraint (Fig. 4). The difference in energy between the two steps is proportional to the difference in total area, which in turn is simply given by the area of the rhomb composed of the two edges prior to and after the move (see Fig. 4). This way each step takes only $\mathrm{O}(1)$ operations. The move is subsequently accepted or rejected according to the Metropolis criterion.

The initial configuration is a stretched, regular polygon. This initial condition and the dynamics defined above imply that the polygon angles are restricted to change in discrete quanta of $\pm 2 \pi / N$. Thus, although the algorithm is off-lattice, the simulated ring is a discrete variant of a freely jointed chain which strictly coincides with the freely jointed model only for $N \rightarrow \infty$.

The simulations were performed for $N$ between 50 and 3200 , and for $p$ between 0 and $4 p_{\mathrm{c}}$. Away from the critical point, the number of steps required for equilibration is $O\left(N^{3}\right)$, but near $p_{\mathrm{c}}$ the simulation length must be extended due to critical slowing down 21. This limited our investigation of the transition to $N \lesssim 3000$.

\subsection{Results}

Figure [5] shows simulation results for the mean area as a function of pressure for different values of $N$. When $p$ is scaled by $p_{\mathrm{c}} \sim N^{-1}$ and $\langle A\rangle$ by $N$ (below $p_{\mathrm{c}}$ ) or by 

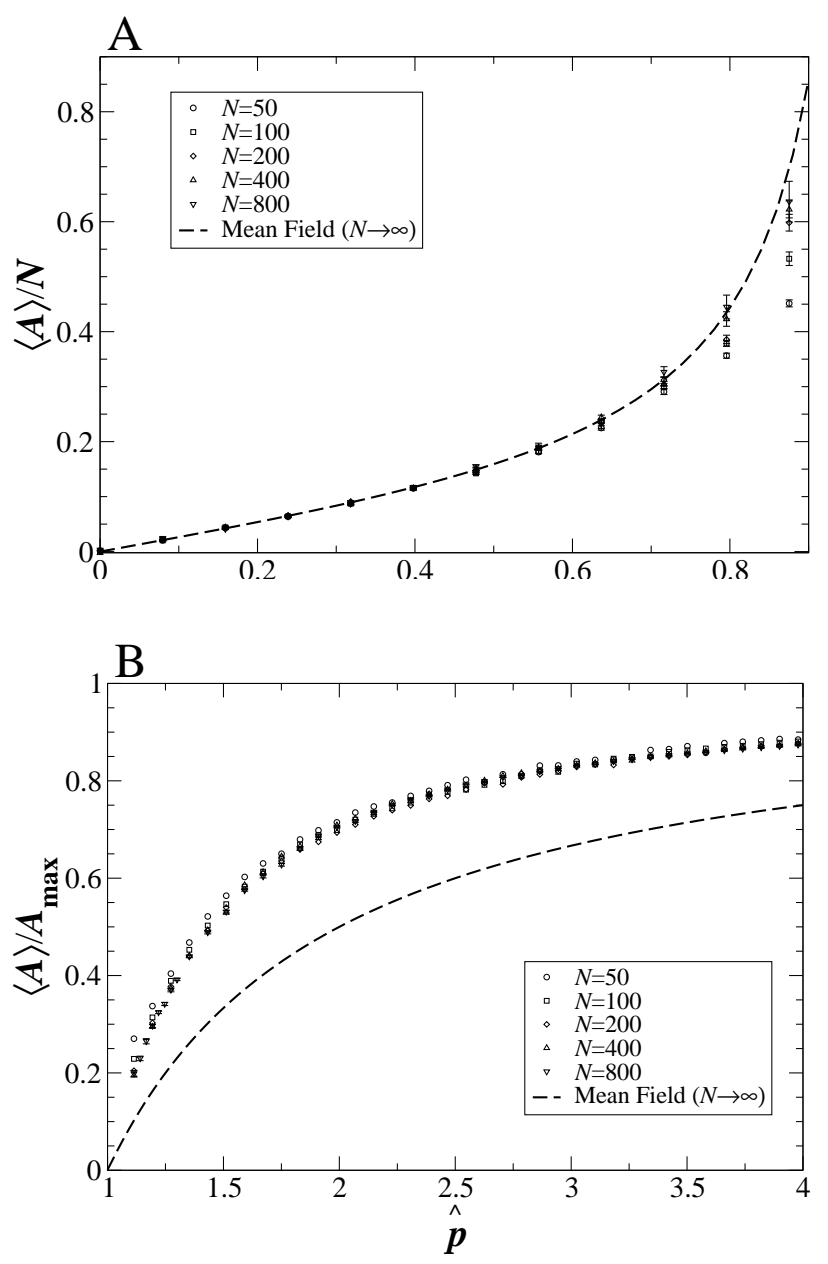

Fig. 5. Mean area as a function of pressure below (A) and above (B) the critical point. The pressure is scaled by $p_{\mathrm{c}}=$ $4 \pi / N$, and the area by $N$ in (A) and by $A_{\max }=N^{2} /(4 \pi)$ in (B). Symbols show the results of MC simulations for different values of $N$. The dashed lines show the prediction of the meanfield theory in the limit $N \rightarrow \infty$.

$A_{\max } \sim N^{2}$ (above $p_{\mathrm{c}}$ ), the data below and above the transition collapse onto two universal curves, thus confirming the predicted scaling laws for the crumpled and smooth states (see Sections 3 and 4). However, while the data well below $p_{\mathrm{c}}$ coincide with the scaling function obtained from the mean-field approximation, the data above the critical pressure collapse onto a different curve.

The simulation results for the mean area and compressibility at $p=p_{\mathrm{c}}$ as a function of $N$ are shown in Fig. 6A. The reduced compressibility, defined in Eq. (18), was calculated from the measured mean-square area fluctuation as $\kappa=(4 \pi / N)\left\langle\Delta A^{2}\right\rangle /\langle A\rangle$. We get

$$
\begin{aligned}
\left\langle A\left(p=p_{\mathrm{c}}\right)\right\rangle & =(0.102 \pm 0.007) N^{1.49 \pm 0.01} \\
\kappa\left(p=p_{\mathrm{c}}\right) & =(0.56 \pm 0.09) N^{0.495 \pm 0.025}
\end{aligned}
$$

Hence, the mean-field exponents for the scaling with $N$, Eqs. (16) and (19), are confirmed. The predicted prefactor of the compressibility is within the standard error of the fit while that of the mean area differs by 4 standard errors.

We also measured from simulations the dependence of the mean-square radius of gyration on $N$, as depicted in Fig. 6B. As predicted, below the critical pressure we find $\left\langle R_{\mathrm{g}}^{2}\right\rangle \sim N^{1.01 \pm 0.02}$, and above it $\left\langle R_{\mathrm{g}}^{2}\right\rangle \sim N^{1.985 \pm 0.011}$. At $p=p_{\mathrm{c}}$ we get

$$
\left\langle R_{\mathrm{g}}^{2}\left(p=p_{\mathrm{c}}\right)\right\rangle=(0.043 \pm 0.025) N^{1.46 \pm 0.13} .
$$
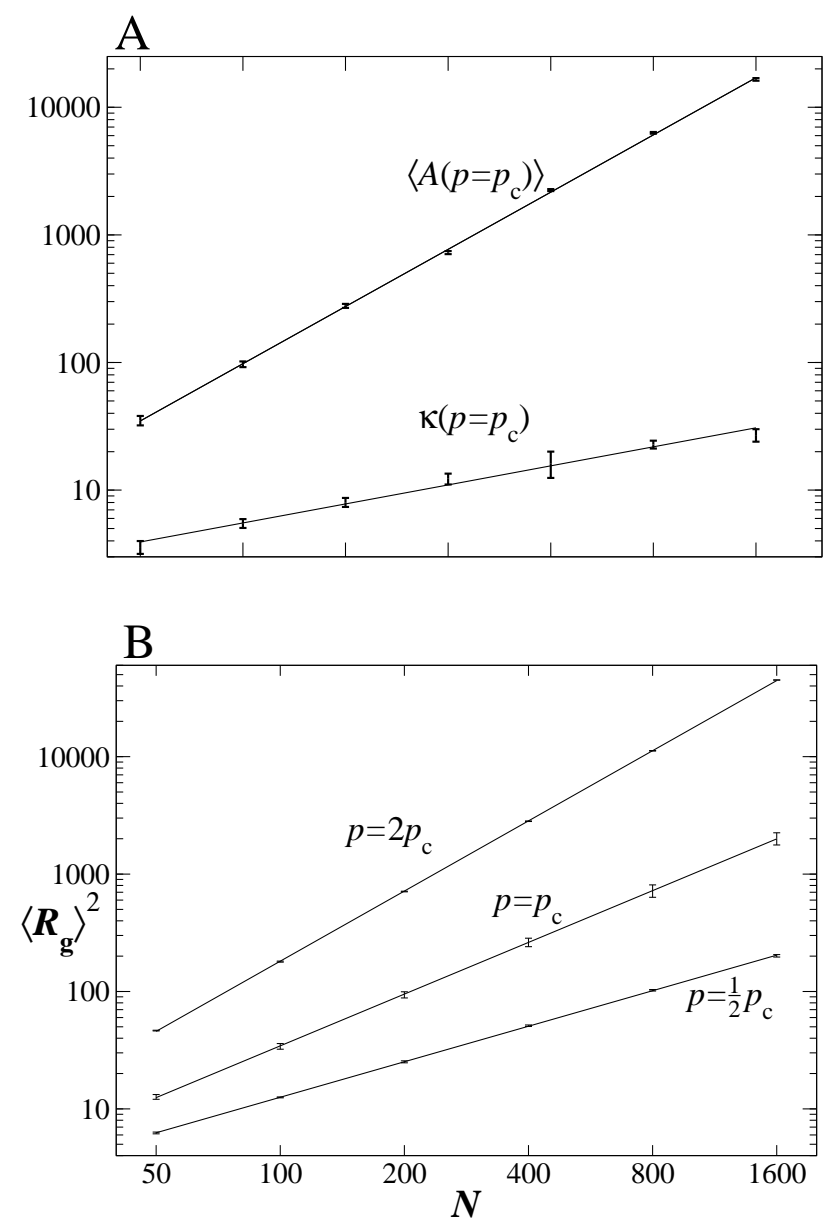

Fig. 6. (A) Mean area and compressibility at $p=p_{\mathrm{c}}$ as a function of $N$ obtained by MC simulations. The fits, $\left\langle A\left(p_{\mathrm{c}}\right)\right\rangle=$ $(0.102 \pm 0.007) N^{1.49 \pm 0.01}$ and $\kappa\left(p_{\mathrm{c}}\right)=(0.56 \pm 0.09) N^{0.495 \pm 0.025}$ are given by the solid lines. (B) Mean-square radius of gyration as a function of $N$ at different values of $p=\frac{1}{2} p_{\mathrm{c}}, p_{\mathrm{c}}$ and $2 p_{\mathrm{c}}$ with the best fits (solid lines) $\left\langle R_{\mathrm{g}}^{2}\left(\frac{1}{2} p_{\mathrm{c}}\right)\right\rangle=(0.12 \pm$ $0.01) N^{1.01 \pm 0.02},\left\langle R_{\mathrm{g}}^{2}\left(p_{\mathrm{c}}\right)\right\rangle=(0.043 \pm 0.025) N^{1.46 \pm 0.13}$, and $\left\langle R_{\mathrm{g}}^{2}\left(2 p_{\mathrm{c}}\right)\right\rangle=(0.019 \pm 0.001) N^{1.985 \pm 0.011}$.

For illustration we show in Fig. 7 four randomly chosen conformations of an 1600-segment ring at the critical pressure. The shapes vary in size significantly due to critical fluctuations. 


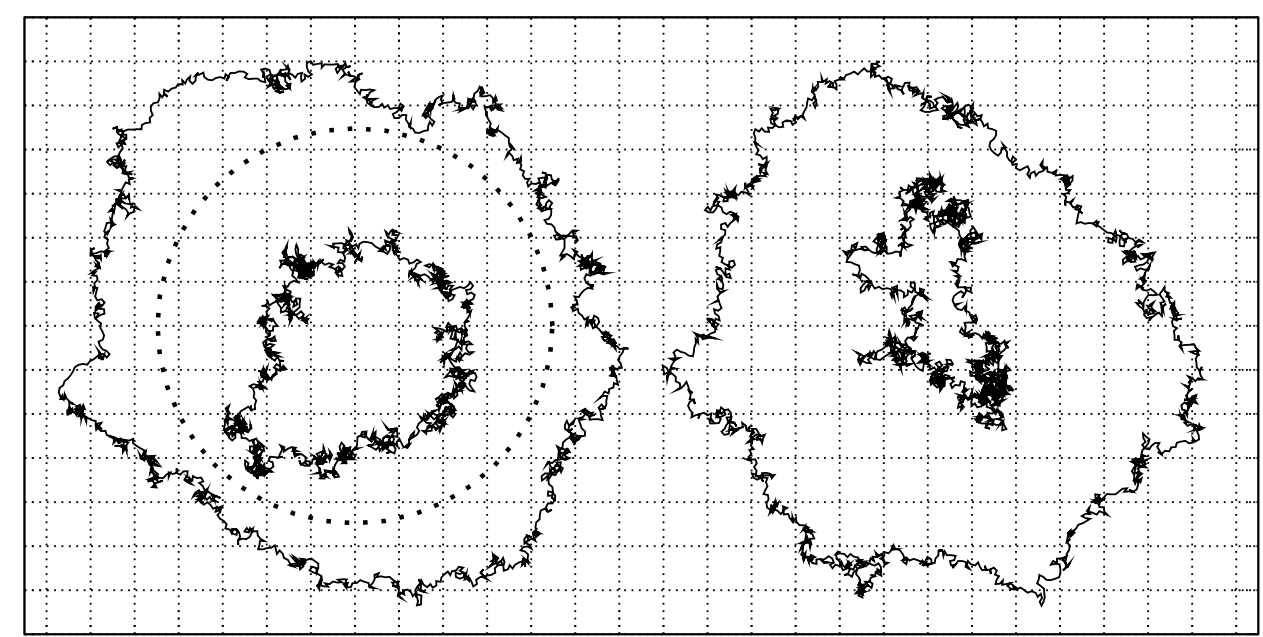

Fig. 7. Four random conformations of a ring with $N=1600$ at the critical state as obtained by MC simulations, demonstrating the critical fluctuations. (The positions of the rings have no significance.) The spacing between gridlines is ten times the link length. The dotted circle, shown for reference, has an area equal to the mean area of the ring at this state.

The simulation results for the smooth state, $p>p_{\mathrm{c}}$, are shown in Fig. 8 where they can be compared with the transfer-matrix calculation, Eq. (26), and the mean-field result, Eqs. (10) and (11). On the one hand, there is good agreement with the transfer-matrix calculation for $\hat{p} \gtrsim 5$, particularly compared to the mean-field result. On the other hand, the mean-field theory succeeds in reproducing the phase transition, whereas the zeroth-order transfermatrix calculation is invalid for these low pressures.

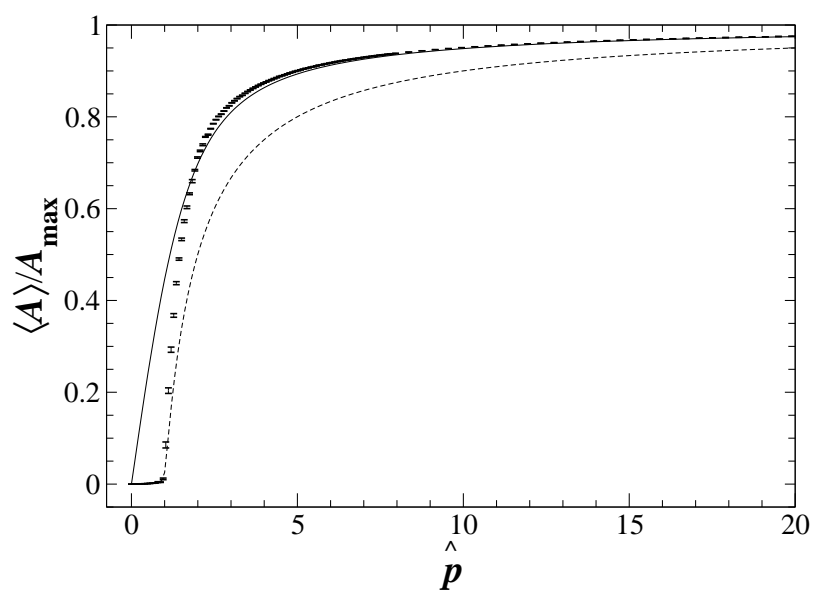

Fig. 8. Mean area (scaled by $A_{\max }$ ) as a function of pressure (scaled by $p_{\mathrm{c}}$ ), as obtained by the zeroth-order transfer-matrix calculation (solid curve), mean-field approximation (dashed curve), and MC simulations for $N=1600$ (error bars).

\subsection{Critical Exponents}

We have confirmed the predicted exponents relating $\langle A\rangle$, $\left\langle R_{\mathrm{g}}^{2}\right\rangle$ and $\kappa$ at $p=p_{\mathrm{c}}$ with the system size $N$. We now turn to the exponents characterizing the divergence with $|\hat{p}-1|$. As in any continuous phase transition, the critical fluctuations make it hard to accurately measure these critical exponents from simulations. Instead, we choose the more reliable route of finite-size scaling to obtain the relations between them.

Let us divide the ring at the critical state into Pincus correlation blobs [17,22] of $g$ monomers and diameter $\xi$ each, such that within a blob the polymer behaves as an unperturbed random chain, i.e., $g \sim \xi^{2}$, whereas the chain of blobs is stretched by the pressure. The perimeter of the ring is $R \sim(N / g) \xi \sim N / \xi$. We have already established that the perimeter of the ring at $p=p_{\mathrm{c}}$ scales as $R \sim$ $N^{3 / 4}$. (See Fig. 6B.) Thus, for a finite-size system, we get a correlation length which scales at $p=p_{\mathrm{c}}$ as

$$
\xi\left(p=p_{\mathrm{c}}\right) \sim N^{1 / 4} .
$$

On the other hand, close to the critical point the correlation length diverges as $\xi \sim|\hat{p}-1|^{-\nu}$, the compressibility as $\kappa \sim|\hat{p}-1|^{-\gamma}$, and the order parameter increases as $M \sim|\hat{p}-1|^{\beta}$. Using Eq. (31) and the numerically established results, $M=R / N \sim N^{-1 / 4}$ and $\kappa \sim N^{1 / 2}$, we obtain the relations

$$
\beta=\nu, \quad \gamma=2 \nu
$$

which hold for the mean-field universality class. (Note that the exponents $\alpha$ and $\delta$ are irrelevant for this system, since both the ordering field and temperature are incorporated in the single parameter $p$.) 


\section{Discussion}

We have demonstrated that the swelling of a 2D freely jointed ring due to a pressure differential exhibits a secondorder smoothening transition of the mean-field universality class. Below the critical pressure the ring behaves as a random walk, with both the mean area $\langle A\rangle$ and mean-square radius of gyration $\left\langle R_{\mathrm{g}}^{2}\right\rangle$ proportional to $N$. In this crumpled state the mean area obeys the scaling $\langle A\rangle=N f_{A}^{<}\left(p / p_{c}\right)$. Mean-field theory accurately captures the scaling law as well as the scaling function $f_{A}^{<}$. (See Fig. 5A.) This lies in the fact that, for an unstretched chain, the Gaussian-spring description in the mean-field calculation and the actual freely jointed model coincide as $N \rightarrow \infty$.

Above the critical pressure $\langle A\rangle$ and $\left\langle R_{\mathrm{g}}^{2}\right\rangle$ are proportional to $N^{2}$. For this smooth state we have found a new scaling behavior, $\langle A\rangle=N^{2} f_{A}^{>}\left(p / p_{\mathrm{c}}\right)$ (Fig. [5 B). The meanfield theory correctly gives the scaling law, yet fails to predict the correct scaling function $f_{A}^{>}$. This is because in a stretched state the entropy of a chain of Gaussian, variable springs is much larger than that of a freely jointed chain of rigid links. For $p \gg p_{\mathrm{c}}$ we have calculated $f_{A}^{>}$ exactly [Eq. (26) and Fig. 8].

At the transition point itself a new state of polymer chains has been discovered, with $\langle A\rangle$ and $\left\langle R_{\mathrm{g}}^{2}\right\rangle$ proportional to $N^{2 \nu_{\mathrm{c}}}, \nu_{\mathrm{c}}=3 / 4$. The swelling exponent $\nu_{\mathrm{c}}$ turns out to be identical to that of a $2 \mathrm{D}$ self-avoiding walk, although the physical origins of the two exponents are unrelated; the freely jointed ring in this intermediate state between crumpled and smooth behaviors contains numerous intersections, as is clearly seen in Fig. 7.

Our discussion has been restricted to freely jointed, self-intersecting chains. We have commented in Section 3 that the addition of self-avoidance to the Flory free energy removes the second-order transition. Thus, the current work suggests a different perspective for the previously studied swelling behavior of $2 \mathrm{D}$ self-avoiding rings 6 6.7. The large-inflation scaling regime thoroughly discussed in those works can be viewed as a broadening of a phase transition present in the non-self-avoiding model, the self-avoidance thus acting as a relevant parameter for the critical point discussed in the current work.

One of the strongest assumptions underlying our analysis, as well as those of Refs. 9, 10,11, is the replacement of the actual geometrical area of the ring with its algebraic area. An important issue is how this assumption affects our results concerning the transition. It is clear that negative contributions to the algebraic area are significant in the crumpled, random-walk state and insignificant in the smooth state. The key question, therefore, is whether they are statistically significant at the transition. Returning to Fig. 7 and the blob analysis presented in Section 6.3 we infer that the negative area contributions lie within the correlation blobs. We have shown that each blob contains $g \sim \xi^{2} \sim N^{1 / 2}$ monomers. The deviation of the geometrical area of a blob from its algebraic area is $\left\langle a^{2}\right\rangle^{1 / 2} \sim \xi^{2} \sim N^{1 / 2}$. (Recall that the blob contains an unperturbed chain with zero mean algebraic area.) The ring contains $n=N / g \sim N^{1 / 2}$ such blobs. Hence, the total deviation of the geometrical area from the algebraic one is $\left(n\left\langle a^{2}\right\rangle\right)^{1 / 2} \sim N^{3 / 4}$. In the limit $N \rightarrow \infty$ this is negligible compared to the mean area of the ring at the critical state, $\langle A\rangle \sim N^{3 / 2}$. Thus, we conjecture that the same smoothening transition as the one reported here will be found also in a model which considers the geometrical area rather than the algebraic one.

We thank M. Kroyter, H. Orland, P. Pincus and T. Witten for helpful discussions. This work was supported by the USIsrael Binational Science Foundation (Grant no. 2002-271).

\section{Appendix: 2D Freely Jointed Chain under Ten- sion}

In this Appendix we recall the results for the partition function of a $2 \mathrm{D}$, open, freely jointed chain subject to a tensile force. These results are used in Sections 3 and 5

Consider a 2D freely jointed chain composed of $N$ links of length $l \equiv 1$. A chain configuration is defined by $N 2 \mathrm{D}$ unit vectors, $\left\{\mathbf{u}_{j}\right\}_{j=1, \ldots, N}$, specifying the orientations of the links. One end of the chain is held fixed while the other is pulled by a force $\mathbf{f}$ (measured in units of $k_{\mathrm{B}} T / l$ ). The partition function of the chain is

$$
\begin{aligned}
Z(\mathbf{f}, N) & =\int \prod_{j=1}^{N} d \mathbf{u}_{j} e^{\mathbf{f} \cdot \mathbf{u}_{j}} \\
& =\left(\int_{0}^{2 \pi} d \theta e^{f \cos \theta}\right)^{N}=\left[2 \pi I_{0}(f)\right]^{N}
\end{aligned}
$$

The mean end-to-end vector is obtained from Eq. A.1 as

$$
\mathbf{R}=\nabla_{\mathbf{f}} \ln Z=N \frac{I_{1}(f)}{I_{0}(f)} \hat{\mathbf{f}} .
$$

The mean end-to-end distance in the limit of weak force, to two leading orders, is

$$
R / N \simeq f / 2-f^{3} / 16
$$

leading, upon inversion, to

$$
f \simeq 2 R / N+(R / N)^{3}
$$

Finally, the free energy for fixed end-to-end distance (to two leading orders in small $R$ ) is

$$
F(R)=-\ln Z[\mathbf{f}(\mathbf{R})]+\mathbf{f} \cdot \mathbf{R} \simeq \frac{R^{2}}{N}+\frac{R^{4}}{4 N^{3}} .
$$

This yields the usual Gaussian term, $F_{\text {el }}$, and the first correction due to inextensibility, $F_{\text {inext }}$, used in Eq. (4).

\section{References}

1. M.G. Brereton, C. Butler, J. Phys. A 20, 3955 (1987).

2. D.C. Khandekar, F.W. Wiegel, J. Phys. A 21, L563 (1988); J. Phys. France 50, 263 (1989).

3. B. Duplantier, J. Phys. A 22, 3033 (1989).

4. B. Duplantier, Phys. Rev. Lett. 64, 493 (1990).

5. J. Cardy, Phys. Rev. Lett. 72, 1580 (1994). 
6. S. Leibler, R.R.P. Singh, M.E. Fisher, Phys. Rev. Lett. 59, 1989 (1987).

7. A.C. Maggs, S. Leibler, M.E. Fisher, C.J. Camacho, Phys. Rev. A 42, 691 (1990).

8. C.J. Camacho, M.E. Fisher, Phys. Rev. Lett. 65, 9 (1990).

9. J. Rudnick, G. Gaspari, Science 252, 422 (1991).

10. G. Gaspari, J. Rudnick, A. Beldjenna, J. Phys. A 26, 1 (1993).

11. E. Levinson, Phys. Rev. A 45, 3629 (1992).

12. U.M.B. Marconi, A. Maritan, Phys. Rev. E 47, 3795 (1993).

13. G. Gaspari, J. Rudnick, M. Fauver, J. Phys. A 26, 15 (1993).

14. A. Dua, T.A. Vilgis, Phys. Rev. E 71, 21801 (2005).

15. Y. Kantor, D.R. Nelson, Phys. Rev. Lett. 58, 1774 (1987); Phys. Rev. A 36, 4020 (1987).

16. E. Guiter, F. David, S. Leibler, L. Peliti, Phys. Rev. Lett. 61, 2949 (1988).

17. M. Rubinstein, R.H. Colby, Polymer Physics (Oxford University Press, Oxford, 2003).

18. R.A. Harris, J.E. Hearst, J. Chem. Phys. 44, 2595 (1966); 46, 398 (1967).

19. B.Y. Ha, D. Thirumalai, J. Chem. Phys. 103, 9408 (1995); e-print cond-mat/9709345

20. R.P. Feynman, A.R. Hibbs, Quantum Mechanics and Path Integrals (McGraw-Hill, New-York, 1965).

21. K. Binder, Topics in current physics, Monte Carlo Methods in Statistical Physics (Springer-Verlag, Berlin, 1979).

22. P. Pincus, Macromolecules 9, 386 (1976). 